SJ Quinney College of Law, University of Utah Utah Law Digital Commons

Utah Law Faculty Scholarship

Utah Law Scholarship

2017

\title{
Frontiers in Precision Medicine II: Cancer, Big Data and the Public
}

Emily Coonrod

University of Utah

Jorge L. Contreras

S.J. Quinney College of Law, University of Utah, jorge.contreras@law.utah.edu

Willard Dere

University of Utah

Jeffrey Botkin

University of Utah

Leslie Francis

S.J. Quinney College of Law, University of Utah, leslie.francis@law.utah.edu

See next page for additional authors

Follow this and additional works at: https://dc.law.utah.edu/scholarship

Part of the Health Law and Policy Commons, Medical Humanities Commons, Oncology Commons, and the Translational Medical Research Commons

\section{Recommended Citation}

Coonrod, Emily; Contreras, Jorge L.; Dere, Willard; Botkin, Jeffrey; Francis, Leslie; and Tabery, Jim, "Frontiers in Precision Medicine II: Cancer, Big Data and the Public" (2017). Utah Law Faculty Scholarship. 74.

https://dc.law.utah.edu/scholarship/74

This Article is brought to you for free and open access by the Utah Law Scholarship at Utah Law Digital Commons. It has been accepted for inclusion in Utah Law Faculty Scholarship by an authorized administrator of Utah Law Digital Commons. For more information, please contact valeri.craigle@law.utah.edu. 


\section{Authors}

Emily Coonrod, Jorge L. Contreras, Willard Dere, Jeffrey Botkin, Leslie Francis, and Jim Tabery 


\title{
FRONTIERS IN PRECISION MEDICINE II: CANCER, BIG DATA AND THE PUBLIC
}

\author{
December 1-2, 2016

\section{Conference Proceedings}
Emily Coonrod, Jorge L. Contreras, Willard Dere, Jeffrey Botkin, Leslie P. Francis, James Tabery ${ }^{1}$

In a December 2016 interview with Bloomberg BNA, Dr. Francis Collins, Director of the U.S. National Institutes of Health, predicted that precision medicine "is going to change everything about how we understand health and disease." ${ }^{2}$ The massive Precision Medicine Initiative (PMI), now re-named the "All of Us" Research Program, plans to study a cohort of more than one million Americans. Its hope is to expand our understanding of heredity and disease and revolutionize the treatment of disease and the improvement of human health.

According to the White House, precision medicine is "health care tailored for you." ${ }^{3}$ At its most fundamental level, precision medicine seeks to optimize treatments based on individual physiological characteristics, such as the ability to metabolize certain compounds or to respond to one or another set of drugs based on individual differences in genes, environments, and lifestyles.

Precision medicine is being developed today within a complex landscape of medicine, science, public policy, law, and ethics. In December 2016, the University of Utah School of Medicine, Huntsman Cancer Institute and S.J. Quinney College of Law held their second annual symposium on current issues in precision medicine. This year, the focus of symposium was on cancer and how precision medicine can enlist "big data" to combat what has been termed "the emperor of all maladies" -- cancer. ${ }^{4}$

This two-day event convened national experts in genetics, medicine, bioinformatics, intellectual property, health communications and bioethics to discuss and debate many of the pressing questions raised by precision medicine

\footnotetext{
${ }^{1}$ The authors thank Jacqueline Etsy Morrison and Jessica Van Wagoner for their assistance in the preparation of this report, and acknowledge Julie Kiefer, Kristina Monty, Jacqueline Etsy Morrison, Melinda Rogers, Samantha Weeks, Dana Wilson, Jonelle White and the 2016-17 Fellows of the Center for Law and Biomedical Sciences for their invaluable support in planning and executing this conference.

2 Jeannie Baumann, NIH's Collins: Precision Medicine Will Change 'Everything', BNA Life Sciences Law \& Indus. Report, Jan. 6, 2017.

${ }^{3}$ White House. The Precision Medicine Initiative, https://www.whitehouse.gov/precision-medicine.

${ }^{4}$ Siddhartha Mukherjee, The Emperor of All Maladies: A Biography of Cancer (New York: Scribner, 2010).
} 
as it relates to cancer research. ${ }^{5}$ The key arguments, conclusions and unresolved issues that emerged from the symposium are summarized in these proceedings. Speakers and participants are referred to by name in the text, with the full conference agenda and speaker names and affiliations in the Appendix.

\section{Tackling Cancer With Precision Data}

\section{a. The Science Of Precision Oncology}

The first session of the Frontiers in Precision Medicine II: Cancer, Big Data and the Public conference hosted by the University of Utah explored issues facing successful clinical implementation of precision medicine in oncology. Ana Maria Lopez introduced this session and kicked off the symposium. She described the elements that will ensure the success of precision medicine including a team science approach, data sharing policies, insurance coverage, and inclusive patient engagement. She stressed the importance of patient engagement and warned that without it, clinical outcomes for diverse communities may worsen and health disparities may grow. When an inclusive approach to precision medicine is taken, these concepts can be applied to public health to stratify risk at the population level and improve prevention and treatment with targeted strategies that will ultimately lead to disease prevention, health promotion, and health inequity reduction.

Following Dr. Lopez's introduction, Kathleen Cooney provided an overview of precision oncology and discussed specific approaches to its implementation in clinical care. Dr. Cooney noted that cancer is a common disease with 1.7 million newly diagnosed cases per year and is responsible for 600,000 deaths per year in the United States. In addition, the cost of cancer care is predicted to balloon to $\$ 170$ billion per year by 2020 due to an increasingly aging population and the increasing costs of new cancer therapies. The evolving recognition of cancer as a genetic disease that can be caused by both germline mutations (mutations in every cell of the body that can be passed on to one's children) and somatic mutations (mutations that develop in certain cells in the body that are not passed on to one's children) has made the practice of precision oncology a reality. The practice of precision oncology is reliant on new technology that rapidly generates sequencing data on all genes in tumor and non-tumor cells from cancer patients. Dr. Cooney pointed out that early successes with therapies targeted to specific mutations serve as a proof of principle that this approach works, although responses are not always sustained. In addition, these therapies are expensive,

\footnotetext{
${ }^{5}$ Video recordings of the entire symposium, as well as slide presentations made by certain speakers, can be accessed at http://law.utah.edu/projects/precision-medicine/frontiers-inprecision-medicine-2016-cancer-big-data-and-the-public/. Proceedings of the University of Utah's first annual precision medicine symposium, "Frontiers in Precision Medicine: Exploring Science and Policy Boundaries" can be found at https://papers.ssrn.com/sol3/papers.cfm?abstract_id=2851946.
} 
have unique side effects, and can contribute to the development of drug resistant tumors. These discoveries demonstrate the significant impact that the implementation of precision oncology can have on both individual patients and public health, as discussed earlier by Dr. Lopez.

Although targeted therapies can be successful, they have uncovered new challenges to patient care. Without a systematic approach to identifying actionable mutations, many may be unable to access new therapies. The Princess Margaret IMPACT/COMPACT trial, a large study with 1,640 participants, revealed that only $15 \%$ of patients in the study were ultimately enrolled in clinical trials and only $5 \%$ were enrolled in clinical trials for a drug that was targeted to their specific genetic diagnosis. ${ }^{6}$ This study made clear that routinely matching therapies to actionable mutations is another hurdle to overcome in the implementation of precision oncology in the clinical setting.

Dr. Cooney described a multi-disciplinary, team approach to precision oncology, the MI-ONCOSEQ (Michigan Oncology Sequencing Center). In this model, the patient first meets with both a medical oncologist and a genetic counselor and provides informed consent. A biopsy of the tumor is then obtained along with a saliva or blood sample. DNA is extracted from the biological specimens and sequenced. Genomic data are analyzed and a Precision Medicine Tumor Board meets to determine if results can inform the patient's clinical treatment and if there are additional findings that will impact the patient's health. The Precision Medicine Tumor Board is composed of experts in clinical oncology, genomics, bioinformatics, pathology, clinical genetics, and bioethics. A patient advocate serves on this Board to ensure inclusion of the patient perspective in the decision-making process. Results are returned to the patient by both the genetic counselor and the medical oncologist. Dr. Cooney then illustrated success stories from the MI-ONCOSEQ project describing patients with medically actionable mutations that responded extremely well to the targeted, molecularly matched treatment recommended by the Tumor Board. In summary, although the genetic cause of cancer can now be identified, doing so requires a multidisciplinary, team based approach.

Dr. Scott Tomlins closed the session with a presentation exploring the "hype versus reality" of precision oncology and reinforced some of Dr. Cooney's comments. Dr. Tomlins noted that identifying medically actionable mutations in cancer patients is not sufficient to drive clinical choices for cancer care. What really matters when moving precision oncology to the clinic is determining how many patients are enrolled in clinical trials based on their sequencing results and how many of these patients have a favorable response to therapy. Dr. Tomlins used the same example mentioned by Dr. Cooney from the IMPACT/COMPACT

\footnotetext{
${ }^{6}$ Tracey L. Stockley et al. Molecular profiling of advanced solid tumors and patient outcomes with genotype-matched clinical trials: the Princess Margaret IMPACT/COMPACT trial, 8 Genome Medicine 109, Oct. 25, 2016.
} 
trial $^{7}$ to illustrate that not every patient is benefitting from sequencing and that the field needs to study further the clinical utility of sequencing for patient care and clinical outcomes. Some large studies are beginning to tackle this by enrolling patients at multiple centers around the country and matching patients with a specific drug based on their genetic results. In this approach, patients are not treated by the anatomical type of cancer they have (e.g., lung, colon, breast), but rather by the mutation causing their cancer. Although this approach will get us closer to understanding patient outcomes, there are still barriers to doing this on a very large scale. The cost of sequencing a large number of people is still high and new therapies are approved for treatment of a specific type of cancer rather than a molecular signature, meaning that pharmaceutical companies will encounter regulatory barriers to drug approval using this approach. Dr. Tomlins also pointed out that there are a number of genes known to cause a high percentage of cancers that have been recalcitrant to therapeutic targeting, such as TP53, KRAS, MYC, APC, CTNNB1, and PIK3CA. New approaches to drug development will be necessary to target the genes that are responsible for many cancer cases. Lastly, Dr. Tomlins predicts that immunotherapy will have the most impact on clinical outcomes because sequencing allows for the creation of an antigen specific to the patients' tumor that will be attacked by their own immune system. To summarize, Dr. Tomlins urges the field to move beyond sequencing to understand clinical utility. This requires improving enrollment of patients into matched clinical trials and systematically measuring outcomes.

\section{b. The Challenges Of Big Data}

New and innovative informatics approaches are necessary to unlock the promise of precision medicine in clinical practice, but working with the large datasets produced by next generation sequencing poses a number of challenges being tackled at institutions around the country. Dr. Rakesh Nagarajan described the convergence of three major innovations that have led to the rapid emergence of precision medicine. First is the development of the technology to do massively parallel sequencing with a cost that continues to decrease. Second is a dramatic increase in medical knowledge-bases enables the interpretation of this sequencing data to inform clinical practices, such as prescribing targeted drugs and immunotherapies. Third are advances in information management and informatics that are bridging the production of sequencing data and medical knowledge to make precision medicine possible. There remain a number of barriers to truly conducting precision medicine at scale to sustain the current exponential growth of DNA sequencing. At a high level, these barriers include acquisition of the data, data storage solutions, distribution of data on a population scale, and analysis capabilities. ${ }^{8}$

${ }^{7}$ Id.

${ }^{8}$ Zachary D. Stephens et al, Big Data: Astronomical or Genomical? 13 PLoS Biology, July 7 , 2015. 
Changing the infrastructure of the electronic health record (EHR) is one way that informatics is being used to address these barriers. The EHR at Washington University in St Louis School of Medicine alone contains records from $\sim 5.7$ million patients with $\sim 40$ million encounters, and contains $\sim 89$ million text documents with $\sim 48$ million scanned documents. The vast majority of the genomic data that is produced clinically is found in text documents. This creates a major informatics problem because it is difficult to electronically extract information from free text due to inconsistencies in ontologies and vocabulary standards. Therefore, a test result and its details are not recorded in the EHR in a way that can be easily extracted and used to treat the patient. An informatics solution has already been put in place for transferring different types of nongenomic data from disparate sources to the EHR using HL7 messaging to transfer and store demographic information, laboratory results, and medication prescribing information in a standardized way. There are now informatics solutions being developed to achieve the same goal with genomic data. One example is the HL7 specification Clinical Genomics Object Model to transmit genomic results as discrete fields. ${ }^{9}$ However, this standard has not been adopted by EHR vendors due to low demand. Additional file formats being standardized for this purpose are CRAM, BAM/SAM, VCF/BCF and HGVS for variant nomenclature.

Biomedical knowledge-bases are significant resources used by clinical laboratories to classify variants and determine if variants are clinically actionable. These knowledge-bases are continuously expanding. However, in order to be useful, the laboratory and knowledge-base must be using the same human genome build to annotate variants or have the capability to map variants from one genome build to another accurately and automatically. There are guidelines published to organizations such as the American Society of Clinical Oncology and the FDA, along with medical publications and clinical trial studies, but these sources are also free text. Oncology Research Sources such as COSMIC, TCGA, and cBioPortal are available in knowledge-bases to help guide clinical laboratory interpretations, as are clinical variant databases such as ClinVar, Emory, ARUP Laboratories, and Invitae. All of these resources aid clinical interpretation, but pose some of the same challenges described above.

PierianDx has begun to address these problems by creating a network of thirteen laboratories offering somatic cancer panels clinically. This laboratory network is conducting clinical tests with 41 different gene panels having an average panel size of 44 genes. Through this network, PierianDx has been integrating information from the sources described above to create a curated knowledge-base for these network laboratories. Genes on panels offered through

\footnotetext{
${ }^{9}$ Roy Somak, et al, Next-Generation Sequencing Informatics: Challenges and Strategies for Implementation in a Clinical Environment, 140 Arch. Pathol. Lab. Med. 958, Sept. 2016.
} 
this network have been curated with FDA drug label information, National Comprehensive Cancer Network guidelines, clinically relevant interpretations, associated clinical trials, and clinical associations. Creating this community knowledgebase allows PierianDx and its network to aggregate results to better understand the genetic basis of cancers such as mutation frequency by tumor type.

This network approach begins to address some of the current informatics issues, and is already being performed nationally in health care systems such as the VA's Precision Oncology Program, which is an integrated database with clinical, genomic, imaging, and research data processed and analyzed through Natural Language Processing (NLP). ${ }^{10}$ The evolution of these networks will ultimately create a national learning healthcare system, which integrates genomic data, prior patient data, and population health data to deliver the best, most appropriate care possible in the era of genomic medicine. Standards such as FHIR, a data specification that allows sharing of data formats across organizations, and the meaningful use-stage 3 program from the Centers for Medicaid and Medicare Services ${ }^{114}$ requiring electronic delivery of care records when patients transition from one hospital organization to another are major steps toward creating this national network. Current technologies also enable a disruptive approach to current care delivery by enabling patients to drive their own healthcare delivery by having access to their individual, comprehensive electronic medical record and being an active and engaged member of the healthcare delivery team. ${ }^{12}$

The second half of the session highlighted systems put in place at the University of Utah's Huntsman Cancer Institute $(\mathrm{HCl})$ to deliver precision medicine. Samir Courdy described the evolution of the informatics infrastructure at $\mathrm{HCl}$ since 1999. Building the capability for delivery of precision medicine began with a redesign of the IT architecture to enable database technologies and web capabilities for infrastructure applications. The next phase of pipeline development from 2002-2006 focused on building the missing links to enable patient education, research, and delivery of care such as creating a research subject registry and integration with the University of Utah Hospital's Enterprise Data Warehouse (EDW). 2006-2010 was a period of customization, expansion, and increased efficiencies while building the critical infrastructure for NLP. Currently, the infrastructure is being expanded to allow for integration of disparate data sets, collaboration with other institutions, and management of big data. Like Dr. Nagarajan, Mr. Courdy also pointed out that one of the major challenges to integration of genomic data into clinical care is our ability to mine

\footnotetext{
${ }^{10}$ http://www.blogs.va.gov/VAntage/22165/va-precision-oncologyl. Accessed 6/2/17.

11 https://www.cms.gov/Regulations-and Guidance/Legislation/EHRIncentivePrograms/Stage3Medicaid Require.html. Accessed 6/2/17.

12 http://slideplayer.com/slide/9984919/. Accessed 6/2/17.
} 
data from free text. Mr. Courdy predicts that this problem will continue to exist into the foreseeable future, as clinical laboratories are designed to communicate results via free text. However, this problem creates opportunities for innovation and research into NLP and machine learning.

$\mathrm{HCl}$ has created a solution for implementing precision medicine termed the Research Informatics Shared Resource (RISR). This pipeline utilizes both commercial and non-commercial components to create an information network that pulls data from the different testing laboratories used by $\mathrm{HCl}$, primarily Foundation Medicine and the University of Utah's ARUP Laboratories, into a research integration engine allowing this data to be deposited into the EHR, the EDW, and shared with national oncology research networks as appropriate. $\mathrm{HCl}$ is working with testing laboratories to solve the problem of receiving genomic results in free text format. Foundation Medicine and ARUP Laboratories are now providing testing data in PDF, BAM, VCF, and/or XML file formats, and HL7 messaging is being used by Foundation Medicine to deposit test results into the University's EHR. The $\mathrm{HCl}$ team is utilizing commercially available and in-house developed tools for NLP to turn free text reports into discrete, searchable, and structured data elements that can be utilized by HCl's research integration engine.

Dr. David Nix next discussed his team's approach to move clinical genomic samples into a translational research program. There are two types of genomic data received by $\mathrm{HCl}$, data generated on samples through research, and data generated on samples through CLIA laboratories from clinical tests. $\mathrm{HCl}$ recently joined the ORIEN network, which aims to leverage multiple data sources from different institutions to better match patients to targeted treatments. $\mathrm{HCl}$ has implemented the Total Cancer Care protocol, a study whose data is shared with the ORIEN network. This protocol provides a uniform patient consent form, an umbrella IRB at each institution to allow data access across institutions, and has put in place a variety of data sharing mechanisms that allows access to both clinical and molecular data. Foundation Medicine is the preferred clinical testing vendor for $\mathrm{HCl}$, because its data is rich and focused on clinical utility, making it useful for sharing within HCl's research network. Foundation Medicine's tests target clinically actionable genes from tumors in two different gene panel tests that detect short variants (SNVs and INDELs), copy number variants, and select gene rearrangements. Importantly, the data are returned to $\mathrm{HCl}$ in discrete field XML clinical reports and unfiltered BAM file formats. These data are then reprocessed before being moved into Mr. Courdy's pipeline. In order to make these clinical data useful for a research program, it is imperative to pull out all of the information from the tests, rather than accessing only the information that is in the clinical report. Dr. Nix's team has created a pipeline to re-process the data from the unfiltered BAM files to normalize the variant calls from different companies and sources in order to unify the datasets and increase their utility for research. There are a number of challenges inherent in the re-processing that are being addressed. First, the output from the re-processing pipeline does not 
always result in the exact same variants called in the clinical laboratory's reports. This is due to differences in variant calling and annotation between the two pipelines. Therefore, it is critical to develop and maintain best practices for the pipeline, implement as much pipeline automation as possible, and develop NLP tools to extract data from free text clinical reports. Dr. Nix asked the audience to consider using drop down menus and ontologies for phenotypic and clinical data management to help relieve the free text extraction problem.

The pipeline created by $\mathrm{HCl}$ utilizes a mixture of commercial and open source tools, and the team is now focusing on scaling up this pipeline to handle the deluge of additional data that will be coming to $\mathrm{HCl}$ when insurance companies begin to more routinely reimburse for genetic panel testing in oncology, thus creating much larger data sets than are currently being managed.

\section{c. Precision Prevention}

The next session focused on the topic of precision prevention and featured discussions on current approaches in the areas of colorectal cancer and lung cancer. The session was moderated by Dr. Cornelia Ulrich. Dr. Ulrich defined precision prevention as "tailoring of preventive strategies more precisely, for example by genetic markers, molecular markers, or overall risk prediction." Taking this approach to cancer prevention can maximize the use of resources and identify specific prevention approaches for groups that will receive the most benefit. Dr. Ulrich then described her group's work in exploring the use of aspirin in colorectal cancer prevention. Aspirin has been shown to reduce the number of polyps in patients with colorectal cancer, has shown an inverse association with colorectal cancer and other tumor types in epigenetic studies, and the molecular pathway affected by aspirin is known. In addition, long term use (10-14 years) of aspirin at a low dose has been shown to be protective against colorectal cancer. ${ }^{13}$ Although the findings that support the use of aspirin to prevent colorectal cancer are strong, there are major risks to long term aspirin use, including GI symptoms and serious bleeding. One way to mitigate these risks is to tailor the recommendations for long term aspirin use to populations who will benefit the most while reducing the risk of side effects. Dr. Ulrich's group has used pharmacogenomics approaches to uncover a specific genotype that reduces the risk of colorectal cancer upon regular aspirin use and a genotype that does not benefit from long term aspirin use. The group is now working on better understanding the cost effectiveness of using genotype-guided aspirin use as a primary chemoprevention method in colorectal cancer versus standard colonoscopy screening. Dr. Ulrich plans to translate these findings into the clinic to stratify patients at risk for colorectal cancer into groups that will benefit from

\footnotetext{
${ }^{13}$ E. Flossmann, P.M. Rothwell, British Doctors Aspirin Trial \& the UK-TIA Aspirin Trial, Effect of aspirin on long-term risk of colorectal cancer: consistent evidence from randomised and observational studies, 369 Lancet 1603 (2007).
} 
aspirin use and those that will not, and to develop a blood test that would allow people at risk for colorectal cancer and their clinicians to make informed decisions on whether the benefits of long term aspirin use outweigh its risks.

Next, Dr. Ulrike Peters discussed her group's efforts to personalize risk prediction for colorectal cancer. Colorectal cancer is the $3^{\text {rd }}$ leading cause of cancer-related deaths in the US, and, although very effective screening tools already exist, approximately one-third of the eligible US population has not received this screening. In fact, because current guidelines are only based on age and family history, rates of colorectal cancer are on the rise in populations that do not meet screening criteria. The goal of Dr. Peters' group is to create a comprehensive risk prediction model to stratify people in high risk groups for both screening and intervention. To realize this goal, Dr. Peters utilizes the large datasets available through the Genetics and Epidemiology of Colorectal Cancer Consortium (GECCO). GECCO is a consortium of investigators from multiple institutions that aggregates data from studies. GECCO allows Dr. Peters' group to use genetic information coupled with epidemiological and environmental information from these studies to innovate new statistical methods for risk prediction. The risk prediction model being developed takes into consideration the following variables: age, family history of colorectal cancer, sex, a genetic risk score, and an environmental risk score while adjusting for endoscopy. Applying this model to a study cohort of $\sim 8,000$ cases and $\sim 9,000$ controls showed that incorporation of the genetic and environmental risk scores increases their ability to accurately predict colorectal cancer risk. Dr. Peters is also using this risk prediction model to inform the age at which an individual would benefit from screening, thus predicting the appropriate age based on the individual's risk to start receiving the life-saving colorectal cancer screening. The next step in this work is to bring personalized screening into public health practice and clinical care. To do so, the risk prediction modeling will need to be further tested in a community based setting and used to predict earlier stages of disease. In addition, the model needs to be subjected to validation and cost effectiveness studies, culminating in a clinical trial to show its utility in colorectal cancer prevention.

Dr. Marc Lenburg then described his work in the area of precision lung cancer prevention. Lung cancer is the second leading cancer diagnosis and the leading cause of cancer death in the US. However, early detection of lung cancer through screening is known to reduce mortality of this disease. ${ }^{14,15}$ Screening for lung cancer by imaging is the most common technique, but this approach leads to high rates of false positives, and only $4 \%$ of individuals showing abnormal lung masses by imaging actually have lung cancer. A definitive lung cancer diagnosis

\footnotetext{
${ }^{14}$ C.F. Mountain, A new international staging system for lung cancer. 89 Chest $225 \mathrm{~S}$ (1986).

${ }^{15}$ National Lung Screening Trial Research, et al. Reduced lung-cancer mortality with low-dose computed tomographic screening. 365 N. Engl. J. Med 395 (2011).
} 
requires the examination of tissue pathology, which is obtained through an invasive diagnostic procedure such as bronchoscopy, needle biopsy, or surgical resection. Therefore, precision prevention of this disease requires the integration of molecular information to guide the diagnostic workup and prevent unnecessary invasive procedures.

The group's early work in this area utilized expression profiling of genes expressed in bronchial tissue to develop a predictive biomarker. The team analyzed gene expression profiles in samples from normal appearing cells collected during bronchoscopy and identified a panel of 80 genes showing different patterns of gene expression from normal vs. diseased cells. ${ }^{16}$ This approach was validated in a clinical trial ${ }^{17,18}$ and is now utilized clinically to differentiate individuals who only need further monitoring from those who require more invasive diagnostic procedures to definitively identify whether a mass is cancer or not. Dr. Lenburg and his team are now moving toward identifying a tissue source that is even less invasive and costly than collecting bronchial tissue. Their studies have focused on cells from nasal tissue collected through a nasal swab based on the theory that if the entire respiratory tract is affected by lung cancer disease processes, then these processes may be detectable molecularly in a tissue type that is more accessible than bronchial tissue. Current work has identified a molecular signature and has shown that adding the molecular information on top of the clinical information leads to improvements in diagnostic sensitivity. ${ }^{19}$ Taken together, this work is leading to less invasive, more cost-effective mechanisms to detect lung cancer early and accurately to ensure that the people who need treatment are getting it as soon as possible, and those that do not have lung cancer are not being subjected to costly and unnecessary procedures.

\section{Catalyzing Translational Innovation}

Dr. Chris Austin, director of the National Center for Advancing Translational Sciences at NIH (NCATS) addressed the topic of personalized medicine from the perspectives of both key national initiatives and a broader institutional effort at NIH. President Obama's State of the Union address in 2015 introduced the Precision Medicine Initiative as an important platform by which the U.S. could more deeply understand variations in individual responses to

\footnotetext{
${ }^{16}$ A. Spira, et al. Airway epithelial gene expression in the diagnostic evaluation of smokers with suspect lung cancer. 13 Nat. Med. 361 (2007).

${ }^{17}$ G.A. Silvestri, et al. A Bronchial Genomic Classifier for the Diagnostic Evaluation of Lung Cancer. 373 N. Engl. J. Med. 243 (2015).

${ }^{18}$ D.H. Whitney, et al. Derivation of a bronchial genomic classifier for lung cancer in a prospective study of patients undergoing diagnostic bronchoscopy. 8 BMC Med Genomics18 (2015).

19 J.F. Perez-Rogers, et al. Shared Gene Expression Alterations in Nasal and Bronchial Epithelium for Lung Cancer Detection. 109 Jnci-J Natl Cancer I (2017).
} 
therapeutic interventions; gain health insights through discoveries made possible by genetic and genomic advances; help address deficiencies in studying underrepresented populations; and use modern technologies such as mobile sensors to better monitor health status. The original design--to enroll a cohort of one million individuals who would be followed longitudinally over a number of decades-is now being realized as the "All of Us" study. ${ }^{20}$ In addition to the data and information derived from this cohort, there are important goals to improve the culture and environment of clinical research in the US by improving the quality of informed consent; better data sharing; and more assiduous response to participant inquiries and return of clinical data results. One year later, an initiative, the "Cancer Moonshot", to accelerate the progress of discovery in oncology was announced. Its goals are similar, namely to accelerate discovery and improve individual patient care through either better targeted therapies or stratification to enhance preventive testing. Key facets of the initiative include the importance of direct patient involvement; team science and national networks in a variety of disciplines including immunotherapy, overcoming cancer resistance, and systematic approach to childhood cancers.

In many ways, these two initiatives are an extension of the NIH focus on improving translational research-namely getting basic discoveries to the public--and is the remit of NCATS. NCATS has a two-fold goal: making fundamental discoveries to treat diseases, like its efforts in rare diseases that afflict approximately 25 million Americans, and improving the science of translation, namely applying the scientific method of evaluating processes and scientific platforms to improve clinical research. Dr. Austin identified the broader societal problem that NCATS attempts to address as follows: As a biomedical community, we are making major scientific advances at the discovery bench, and due to advances in technology, are poised to make even more discoveries directed to understanding the genetic basis for thousands of rare diseases. Unfortunately, our antiquated processes to translate these basic discoveries to improvements in clinical care greatly delay the possibilities of enhancing the impact of discoveries on broader public health. Thus, Dr. Austin has focused NCATS on improving both the processes and culture of translational research, with full awareness that quantum advances are more important than small incremental improvements. Two such process improvements and innovations include: (1) better utilization of available libraries of small molecules and information about their pharmacology profiles to identify potential treatments for rare diseases; and (2) developing tools better to predict the potential toxicities of potential new therapies.

The CTSA (Clinical and Translational Science Award) program is one of the centerpieces of NCATS's efforts, and supports translational research efforts in approximately 60 academic medical centers. The program seeks to help improve the culture of translational research by rewarding team science and collaboration, in addition to supporting other vital goals such as training the next

${ }^{20}$ https://allofus.nih.gov/. Accessed 11/7/2017 
generation of biomedical scientists, and emphasizing the importance of deep community engagement and patient-centered research. One area of immediate priority is to establish an effective consortium of the CTSA centers (called the Trial Innovation Network) which can readily participate in multi-center clinical trials by making the trial planning and initiation processes more efficient through various measures such as single IRB approval and improved contracting. ${ }^{21}$

\section{The Public And Precision Medicine}

\section{a. Recruiting Large Research Cohorts}

The ambitious plan of the "All of Us" Research Program is to recruit 1,000,000 or more American volunteers who will provide researchers with access to their genetic information, their environmental exposures and activities, and their electronic medical records, all with the aim of understanding how genetic predispositions, environmental exposures, and lifestyle choices contribute to health and disease. This program has been praised for its ambitious size and scope, its tremendous clinical promise, and its daunting set of scientific, technological, and ethical-legal challenges.

The combination of excitement and trepidation surrounding the All of Us Research Program might give some the impression that it is the first effort to create a large, longitudinal research cohort designed to investigate genetic and environmental impacts on health. In fact, however, a number of regional, national, and international programs have been created in the last 15 years with precisely that design and purpose. As the United States embarks on the next national cohort, there are valuable lessons to be learned from others who have already gone down that path. This session sought to gain insights from two researchers who have been intimately involved with efforts in the United States (one public, one private) to create large, longitudinal research cohorts. Laurence Meyer discussed the Million Veterans Program that is housed in the Veterans Administration (VA). Catherine Schaefer described the Research Program on Genes, Environment and Health run by Kaiser Permanente Northern California (KPNC). Challenges, foci, and lessons common to both programs were particularly insightful.

The Million Veterans Program was conceived over a decade ago when researchers at the VA realized that they had access to a tremendous resource in the millions of veterans receiving healthcare through the VA medical system. The VA developed one of the first electronic medical record systems in the world, and was thus in a strong position to link genetic/environmental/lifestyle information about their patients with the electronic medical records of those patients which go back decades. The Research Program on Genes, Environment, and Health was developed by geneticists and epidemiologists at KPNC in the early-2000s. Like

${ }^{21}$ https://ncats.nih.gov/ctsa. Accessed 11/7/2017 
the researchers at the VA, the KPNC researchers realized that the 3.3 million members receiving their healthcare through the health delivery system offered a remarkable potential data set for investigators. Both programs have already enrolled several hundred thousand participants, and both have been utilized for a variety of research projects (e.g. a genome-wide association study of schizophrenia by the VA, and a genome-wide association study of bipolar disorder by KPNC).

There are striking similarities regarding challenges, foci, and lessons faced by these two large, longitudinal cohort programs:

Underrepresentation of Young Adults: Both programs struggled to enroll younger participants (i.e. ages 18-39). The population of the cohorts both skewed older than the U.S. population. The reasons for lower enrollment among younger individuals was a topic of speculation-perhaps younger people are more busy/distracted and so less likely to enroll. Regardless, the underrepresentation among younger participants is a potential concern for cohort designers and researchers. If researchers are interested in investigating conditions with earlyadult onset, then it will be important to ensure there are sufficient representatives from that group to support that research.

Data Management: Both programs encountered significant challenges associated with managing the data from the cohorts (see also the discussion in Part I.b, above). Some of these challenges arose from trying to take the information in electronic medical records, which are designed primarily for the care of patients, and translating/distilling that into standardized information that can be quantified and tracked for research purposes. Other challenges arose from the sheer quantity of data. A single participant can create dozens of data points from a single questionnaire. When that information is combined with an electronic medical record, the number of data points jumps to the thousands. Finally, when genetic information about that participant is figured in, it can become millions of data points. And that's just for one participant. When that is multiplied by the several hundred thousand or million participants in the entire cohort, the amount of data becomes logistically and technologically daunting. The VA found this challenge so severe that it had to team up with the Department of Energy in order to access the computing power necessary to handle the data. That resource, of course, will not be available to all research cohorts.

Genetic Focus: Both cohort programs professed an interest in supporting research on the genetic, environmental, and lifestyle contributions to health and illness. And yet, the design and performance of both programs indicated a stronger genetic focus. When giving examples of research supported by the programs, for example, both Meyer and Schaefer discussed genome-wide association studies. To be clear: this is not to suggest that the researchers themselves have a genetic bias. Rather, it is most likely a result of the fact that 
genetic information is much easier to get and analyze than environmental/lifestyle information. A simple blood or saliva sample can provide material for producing an individual's entire human genome. Information about that same person's environment and their lifestyle, on the other hand, is much more difficult to acquire and assess. The cohort programs at the VA and KPNC both relied in part on questionnaires/surveys administered to their participants to gather information about lifestyle, but those methods are notoriously unreliable. Getting objective data about environmental exposures and lifestyle decisions (with things like wearable monitors, in-home monitoring devices, and geocoded databases that link participants' locations with information about environmental factors in those areas) is more difficult and more expensive. Until measuring the environment in a reliable way becomes easier and cheaper, these large, longitudinal research cohorts will continue to produce more information about genetic contributions to health/disease than environmental/lifestyle contributions to those same conditions.

Early Community Involvement: Both Meyer and Schaefer emphasized the importance of getting input from potential participants at the very earliest stages of cohort creation. The first step in the Million Veterans Program was to ask veterans affiliated with the VA what they would want the cohort to look like. Similarly, the program at KPNC created a community advisory board early on in order to get its advice. Formally implementing this community-input step early is important because it gives potential participants the opportunity to shape the cohort program from its inception-to contribute to decisions about what sorts of research questions the cohort can be designed to answer, what sorts of motivations make participating attractive, and what sorts of concerns about participation disincline someone from enrolling.

The differences between the Million Veterans Program at the VA and the Research Program on Genes, Environment, and Health at KPNC are quite striking. One is a federal program made up entirely of veterans. The other is a private program made up entirely of patients/members affiliated with a national health care delivery system. And yet, both programs encountered similar challenges (of underrepresented young adults, of data management), produced similar research results (focused mainly on genetics), and offered similar lessons (about the importance of involving the community of potential participants early in the decision-making process). The fact that these similarities occurred despite the differences in the programs suggests that they are general features that any large, longitudinal research cohort can face. As the United States embarks on a new, national cohort-the Precision Medicine Initiative's All of Us Research Program-insights from the large, longitudinal cohorts at the VA and KPNC are likely to prove invaluable. 


\section{b. Engaging The Public In The Program}

This session addressed prevalent challenges shared by both the public and professionals with the communication and interpretation of genetic information and the informed consent process. Dr. Kimberly Kaphingst emphasized the prevalence of low health literacy in the general population in the US, noting particular challenges for minority populations, older individuals, and individuals with lower educational attainment. For example, data suggest that almost half of individuals with less than high school or some high school education have a below basic level of health literacy. The implication is that professional efforts to communicate complex information to patients must be sensitive to the low levels of background health literacy that are common in the population. Dr. Kaphingst also highlighted the difficulties and current inadequacies in obtaining informed consent for research such as for biobank participation. The average reading level of the US population is at the $8-9^{\text {th }}$ grade level yet consent forms are often written at a college reading level. The presentation addressed a number of efforts documented in the literature to improve the content and process of informed consent. These include communication strategies such as simplified language, clear organization, descriptive headings, illustrations, and new formats such as videos. Further, she emphasized need to think beyond the form to consider enhanced processes involving better training for professionals, more extended dialogue with patients or research participants, and measures to assess comprehension. Dr. Kaphingst suggested that there will be no easy solutions to these challenges but work along a number of avenues to use and assess better communication strategies will be valuable.

Dr. Angela Fagerlin next addressed a set of challenges in the flow of genetic information between the laboratory, the health professional, and the patient. A particular problem in the current state of genetic knowledge is our inability to provide clear interpretations of many genetic variants identified through testing. A second set of issues arises from the limited knowledge and experience of many health care professionals with respect to ordering and interpreting genetic tests. Data suggest that providers themselves recognize their limits in this respect and have a low level of confidence in their abilities. Individuals who obtain genetic information through DTC sources often choose not to share these results with their physicians because they lack confidence in the ability of their physician to interpret the information. Several measures have been assessed to enhance communication of risk-related results between physicians and patients including different types of graphic presentations. Data indicate that these measures can impact interpretations of risk. Dr. Fagerlin also addressed public attitudes about several significant ethical issues in genetic testing, including the appropriate disclosure of medically actionable results, testing children for adult onset conditions, and the disclosure of carrier status. Data suggest that lay individuals will change their opinions to conform more closely with current professional standards when they are provided information about the ethical concerns in these domains. This session emphasized the significant challenges 
and barriers to precision medicine arising from the background knowledge of both professionals and the public and the need for more effective communication strategies to deal with this complex information.

\section{c. The Ethics of Access and Precision Medicine}

The million lives cohort, as planned for the cancer moonshot and the precision medicine initiative more generally, raises significant issues of justice. These issues will include recruitment of cohort members, informed consent by cohort members, participation of cohort members in decisions about data collection and use, data sharing, communication of findings based on cohort data, and the ultimate availability of benefits from research discoveries. Panelists Jessica Roberts and Dr. Maya Sabatello discussed several of these important issues.

Professor Roberts's presentation considered downstream and upstream access issues. Downstream access issues, such as who will have access to the benefits of the precision medicine initiative are surely important. Benefits such as individualized forms of cancer treatment can be expected to be very costly. But Roberts also emphasized the importance of considering upstream barriers to access. Such barriers might be built into the rules, algorithms, and reference databases that make up the precision medicine initiative, Prof. Roberts said. If the information that is collected from participants in the million lives cohort includes genetic information and clinical information but leaves aside housing, community safety, employment status, environmental exposures, immigration status, or available services, it may focus myopically in medical information and ignore potentially critical roles of the social determinants of health. If certain groups are underrepresented in the research, results pertaining to them may be less accurate as well.

The precision medicine initiative is a very expensive enterprise, Roberts emphasized. It may consume a great deal of research resources and is, itself a "resource allocation" decision. As such, it is especially important to scrutinize diseases to be studied, research questions to be asked, and who is more likely to benefit from the perspective of justice. Prof. Roberts also raised the concern that historical participation in medical research has been skewed by race and ethnicity; it will be important that the precision medicine initiative do a better job of addressing this issue. It will not be easy; African-Americans particularly are less likely to trust participation in research because of the legacy of Tuskegee and the more recent telling of the story of Henrietta Lacks. ${ }^{22}$

Prof. Roberts' takeaways to the audience were first, that access issues in precision medicine reach far beyond who has the benefit of using the end product, to the relative benefits and accuracy of the research for affected

${ }^{22}$ Rebecca Skloot, The Immortal Life of Henrietta Lacks (2010). 
populations, and second, that the initiative must fund research on conditions affecting a variety of populations and build the trust needed to recruit diverse research participants.

Dr. Sabatello's talk addressed issues for persons with disabilities raised by the precision medicine initiative. She began by pointing out that although $22 \%$ of US adults and $13 \%$ of US children have disabilities, disability to date has not received much discussion in the precision medicine initiative.

To the extent that disabilities are genetic in origin, they will be subject to disability rights criticisms of genetic research. These include genetic essentialism and the sorry history of eugenics. Some forms of pre-natal testing, preimplantation genetic analysis, or postnatal predictive and diagnostic testing may reveal discriminatory attitudes such as the devaluation of lives with disabilities or the presentation of unjustifiably negative prognoses. Disability rights critics point to the wide variety of disability and to how the geneticization of impairment may direct attention and budgetary allotments to medical interventions rather than to the social and environmental factors that cause or contribute to disability. Precision medicine has the promise to be attuned to the social model of disability - how social factors contribute to the impact of disabilities on peoples' lives-but only if it is constructed to take these factors into account.

Despite the universality of disability, moreover, people with disabilities continue to experience significant health disparities and disparities in access to care. There are high rates of co-morbidity that are not well understood, such as increased risks of epilepsy and Parkinson's disease among people with intellectual disabilities, and variant cancer rates among people with schizophrenia. Physical barriers such as inaccessible clinic design or diagnostic equipment remain problems. People with disabilities are less likely to participate in clinical trials. They are also less likely to have private health insurance and instead to be dependent on Medicaid or Medicare for payment for their care-or to forego health care instead.

Dr. Sabatello then emphasized the importance of making information about the precision medicine initiative available to people with disabilities. Accessible information about participation, such as publicity in Braille or in plain language, is critical. So is reconsideration of informed consent requirements and processes, so as not to exclude populations of people with disabilities who might not be able to sign or understand standard consent forms. Inclusion of people with psychiatric disabilities may also be an issue from the perspective of informed consent. Another concern will be how to deal with how people change and acquire disability over the long course of the precision medicine initiative. It will be important to consider information and consent as the initiative develops, so that people are not improperly dropped from the cohort or included when they would choose not to continue. 
In sum, the precision medicine initiative will require continued attention to issues of justice, from its beginnings in the questions asked and populations enrolled, to its endpoints in research discoveries and their translation into improvements in patient care and public health. These are not static questions or answers, but will change and develop over time.

\section{The Economics of Precision Medicine}

\section{a. Patenting And Precision Medicine}

Patents have traditionally given the developers of diagnostic and therapeutic products a period of exclusivity (20 years in the United States and most other countries) during which to use those products free from market competition. On one hand, the exclusivity afforded by patent protection enables inventors to charge rates for their products that offset the significant costs of product development and regulatory approval (estimated to be in excess of one billion dollars for a typical pharmaceutical product). On the other hand, the pharmaceutical industry has been criticized for charging excessive rates that make life-saving drugs unaffordable to many, both in the U.S. and abroad (particularly in the developing world). In general, critics of the patent system have alleged that the issuance of too many patents in areas of biomedical research can impede research and scientific progress. ${ }^{23}$

The development of precision medicine therapies has complicated the patent landscape, with recent Supreme Court decisions casting doubt on the degree to which such innovations are eligible for patent protection at all. This panel explored a range of issues relating to the patentability of precision medicine techniques, the potential impact of recent judicial decisions on protection offered by the patent system, the effect of patents on biomedical research at academic institutions and elsewhere, and policy and public health considerations surrounding all of these issues.

Jorge Contreras introduced this session with an overview of the statutory and judicial state of the law surrounding patent eligibility in the United States. Section 101 of the U.S. Patent Act provides that "Whoever invents or discovers any new and useful process, machine, manufacture, or composition of matter ... may obtain a patent therefor." ${ }^{24}$ While this definition can be applied in a relatively straightforward manner to inventions involving new mechanical devices, serious questions of patent eligibility arise when an inventor seeks to claim something derived from the natural world (a so-called "product of nature"), or something that is in essence an abstract idea or mental process. Traditionally, courts have held

\footnotetext{
${ }^{23}$ Mark A. Heller and Rebecca S. Eisenberg, Can patents deter innovation? The anticommons in biomedical research. 280 Science 698 (1998).

${ }^{24} 35$ U.S.C. Sec. 101.
} 
that neither products of nature, abstract ideas nor mental processes are eligible for patent protection.

These patentability exclusions have recently been put to the test in the context of biomedical innovation. Beginning with the seminal case Diamond $v$. Chakrabarty in $1980,{ }^{25}$ the U.S. Supreme Court recognized the patentability of man-made biological organisms (in this case, a bacterium customized to break down crude oil). Based on the Chakrabarty decision, the U.S. Patent and Trademark office (PTO) began to allow patents on biological entities. This practice became particularly controversial in the area of human DNA. Beginning in the early 1990s, researchers began to seek patent protection for newlydiscovered DNA sequences. Among the most controversial of these were patents obtained by Myriad Genetics and the University of Utah on the BRCA1 and BRCA2 genes, as well as certain mutations to those genes that indicated substantially elevated risk for breast and ovarian cancer. The BRCA patents were challenged in a 2009 lawsuit, which alleged that they covered products of nature that were ineligible for patent protection. ${ }^{26}$ In 2013, the Supreme Court invalidated most of the challenged patent claims, holding that human DNA, even if isolated and purified in the laboratory, constituted a product of nature that was not patentable subject matter. ${ }^{27}$

Even more relevant for precision medicine are individualized treatment regimens based on patient physiology and responses. In 2006, three justices of the Supreme Court hinted that they were skeptical of patents claiming such diagnostic methods. ${ }^{28}$ However, it was not until 2012, in Mayo v. Prometheus, ${ }^{29}$ that the Court squarely considered the patentability of a personalized medical treatment. In Mayo, the challenged patent claimed a method of determining the optimal dosage of thiopurine (a drug for an immune-mediated gastrointestinal disorder) by monitoring the level of 6-thioguanine (a metabolite of thiopurine) in a patient's blood after administration of the drug. If metabolite levels were below a certain level, the thiopurine dosage should be increased, and vice versa. The Court, in holding that the claimed subject matter was not patent eligible, reasoned that the relationship between metabolite levels and drug efficacy is a "law of nature", and that a patent should not be granted unless the inventor adds "additional features" beyond that basic natural law. Moreover, the physician's

\footnotetext{
25447 U.S. 303, 317-18 (1980).

${ }^{26}$ For a detailed account of the BRCA litigation see, e.g., Jorge L. Contreras, Narratives of Gene Patenting, 43 FLA. ST. L. REV. 1133 (2016), and Sandra S. Park, Gene Patents and the Public Interest: Litigating Association for Molecular Pathology v. Myriad Genetics and Lessons Moving Forward, 15 N.C. J.L. \& TECH. 519 (2014).

27133 S. Ct. 2107 (2013). The Court also held, however, that man-made DNA segments (e.g., cDNA), even if replicating the sequence of naturally-occurring DNA, were eligible for patent protection.

28 Lab. Corp. of Am. Holdings v. Metabolite Labs., Inc. 548 U.S. 124 (2006) (dissent to denial of certiorari).

${ }^{29}$ Mayo Collaborative Servs. v. Prometheus Labs., Inc., 132 S. Ct. 1289 (2012).
} 
observation of metabolite levels in the blood and decision to alter the patient's thiopurine dosage on that basis, is simply a mental process. Thus, because the claimed invention simply relied on an observation of a natural relationship and a corresponding alteration of the patient's drug dosage, the patent was found to be invalid.

Bernard Chao, in a talk entitled "Measuring the Mayo Effect," began by noting many of the dire predictions made by commentators following the Mayo decision. These commentators predicted that in the wake of Mayo, many personalized medicine technologies would no longer be patentable, thereby causing serious problems for the medical diagnostics industry and potentially reducing innovation in this field. ${ }^{30}$ To test these predictions empirically, Prof. Chao and collaborators measured the number of precision medicine patent applications rejected by the PTO from 2006 through 2016, a ten-year period spanning the issuance of the Mayo decision. Based on a sample of $10 \%$ of the total set of patent applications filed in the relevant technology class, they found that PTO rejection of applications based on Section 101 patent eligibility grounds increased dramatically, from $15.9 \%$ prior to the Mayo case to $86.4 \%$ after Mayo. ${ }^{31} \mathrm{He}$ also described a subsequent study in which the investigators compared Section 101 patent eligibility rejections across ten different technology classes. They found the highest rate of such rejections in the class pertaining to precision medicine. Further studies are being planned to obtain more detailed data on these rejections and their impact.

Colleen Chien also addressed the impact of Mayo and a related 2010 patent eligibility case, Bilski, ${ }^{32}$ in a talk discussing work conducted with Arti Rai of Duke University. Professors Chien and Rai sought to understand the impact of patentability limitations on innovation in the medical diagnostics industry. They found that in the area of biomarker-based diagnostics, patent application filings (which are viewed as proxies for innovation) dipped immediately after the Mayo case, but have recovered since then, suggesting that firms have developed effective strategies for overcoming Section 101 eligibility rejections in this field. Likewise, they found that Mayo had no significant impact on either the size of firms patenting in the area of diagnostics or the number of commercial transactions conducted in the diagnostics industry (which increased sharply after 2012). However, based on measurable increases in the word length of patent claims, they hypothesize that the scope of patent protection in the diagnostics

\footnotetext{
${ }^{30}$ See, e.g., Arti Rai, Biomedical Patents at the Supreme Court: A Path Forward, 66 Stan. L. Rev. Online 111 (2013), Christopher Holman Mayo, Myriad, and the Future of Innovation in Molecular Diagnostics and Personalized Medicine, 15 N.C. J. L. \& TECH. 639, 677 (2014), Rebecca S. Eisenberg, Diagnostics Need Not Apply, 21 B.U. J. SCl. \& TECH. L. 256, 285 (2015).

${ }^{31}$ Bernard Chao and Amy Mapes, An Early Look at Mayo's Impact on Personalized Medicine Patenting, Patently-O blog (April 4 2016), http://patentlyo.com/lawjournal/2016/04/personalizedmedicine-patenting.html. See also Heidi Ledford, US Personalized Medicine Industry Takes Hit from Supreme Court, 536 Nature 382 (2016).

${ }^{32}$ Bilski v. Kappos, 561 U.S. 593 (2010).
} 
field has narrowed since Mayo, as firms have been required to add additional limitations to their patent claims in order to overcome Section 101 rejections. They conclude that, notwithstanding many predictions, innovation in the field of biomarker-based diagnostics has increased, not declined, since Mayo.

Next, Kshitij Kumar Singh offered an international perspective on precision medicine patenting with a particular focus on India. He first noted a number of challenges facing India and other developing countries in the area of healthcare, notably the affordability and accessibility of advanced medical technologies and treatments, the difficulties of a large and disorganized health and insurance sector, and a fragmented research landscape. Given these challenges, coupled with the dominance of foreign firms in the biopharmaceutical industry, policy makers in India have become wary of intellectual property protection for biomedical products. Accordingly, as permitted under the TRIPS Agreement, ${ }^{33}$ the Indian Patent Act prohibits the patenting of "any process for the medicinal, surgical, curative, prophylactic [diagnostic, therapeutic] or other treatment of human beings or any process for a similar treatment of animals to render them free of disease or to increase their economic value or that of their products." ${ }^{34}$ However, he also noted that if an invention includes a novel biomarker or detection method, there may be ways to pursue patent protection. The impact of such policies on both foreign firms seeking to enter markets in developing countries, as well as local innovators in those countries, will be important as policy makers in developing countries continue to refine their local legal structures to address emerging technologies in the field of precision medicine. ${ }^{35}$

In the final presentation of this session, Shubha Ghosh discussed the current policy landscape pertaining to precision medicine, big data and intellectual property. ${ }^{36} \mathrm{He}$ observed the increasing emphasis on data extraction and analysis in the biomedical field, which has been coupled with increasing patenting in this area. He also examined current administration proposals in areas including Medicaid/Medicare reform and competition law enforcement and their potential impact on innovation. He then questioned whether incremental innovation in U.S. precision medicine will be likely in view of the current policy and political landscape. Ultimately, he concluded, incentives must be available to encourage innovation, though these incentives may arise from mechanisms other than patent law.

${ }^{33}$ Article 27.3 (a) gives Member Nations of WTO an option to exclude from patentability "diagnostic, therapeutic and surgical methods for the treatment of humans or animals

${ }^{34}$ Indian Patents Act 1970, amended 2005, Sec. 3(i).

${ }^{35}$ See, generally, Kshitij Kumar Singh, Biotechnology and Intellectual Property Rights: Legal and Social Implications (Springer, 2015).

${ }^{36}$ See, generally, Shubha Ghosh, Identity, Invention, and the Culture of Personalized Medicine Patenting (Cambridge Univ. Press, 2013). 


\section{b. Providers, Payers, and Laboratory Testing}

In the final session, Dr. Diana Brixner introduced the audience to the complex world of reimbursement from a payer perspective. There has been explosive growth in the number of specialty, frequently expensive drugs, which is differentiated from those-generally small molecules or pills-used in primary care for complex chronic disorders. The overall proportion of the pharmaceutical budget devoted to specialty drugs has similarly increased, posing a growing dilemma for payers on how to pay for these drugs through insurance without increasing member premiums. One tool for assessment of these new, expensive drugs is to balance the differential benefit against the differential cost.

Different professional groups are attempting to grapple with measuring "value" of therapeutic interventions being used by practitioners. NCCN (National Comprehensive Cancer Network) and ASCO (American Society of Clinical Oncology) are two major examples for oncology. NCCN provides a grade of 1 (weak) to 5 (strong) for available clinical evidence on key parameters of efficacy; safety; availability of comparative data; consistency of data; and affordability, related to drug-acquisition cost. ASCO utilizes a value framework in evaluating the clinical benefit of cancer treatments against their costs and toxicity. Conceptually, there is an attempt to compare the net benefits (improvement in survival vs. toxicity of therapy) of two comparative regimens to identify the intervention of greater value. Another society-ICER (Institute for Clinical and Economic Review, not to be confused with the same acronym which denotes incremental cost-effectiveness ratio)—seeks to attain sustainable high-value care for all patients. Its value framework evaluates long-term value-for-money assessments based on incremental and comparative cost-effectiveness, and short-term affordability as assessed by budget impact. As information on value become more available, innovative contracting approaches are evolving. For example payers may be reimbursed by the drug manufacturer if specific clinical outcomes are not achieved from the therapeutic intervention, called "outcomesbased contracting". For drug interventions that require a significant initial payment, for example Solvadi in treating and potentially curing hepatitis $\mathrm{C}$, the initial cost can be shared by the initial and future payers. Personalized medicine will play a larger role in defining the health plan population that will receive the greatest benefit from these new therapies.

Payers are also assessing different ways of contracting with health care providers. These include contracting and reimbursing for management of a disease rather for individual drugs

Dr. Christopher Corless outlined the promise that a deeper molecular understanding of disease-for example the new taxonomy of non-small cell lung cancer-and targeted therapies such as ALK-directed medicines are providing to stricken patients. He then delineated the health care provider dilemma: precision medicine and targeted therapies require accurate diagnostics, but development 
and regulatory approval of both the therapy and the diagnostic test are commonly asynchronous, and reimbursement approval is haphazard and insufficient to compensate for the major laboratory investments required to handle biological samples and analyses. Hence cumbersome barriers frequently confront both patient and provider.

A more logical manner of reimbursement in oncology is an urgent need. The burgeoning field of immuno-oncology will require laboratories to evaluate both tumor samples and the patient's own immune system. Furthermore, following cancer patients over time ideally requires both extensive initial evaluation of the tumor and its microenvironment but also metastatic lesions or chemotherapy-unresponsive tumors. Finally, advances in other "-omics" technologies and the increasing data load requires great technical expertise and is costly.

Dr. Craig Nichols concluded with a historical perspective on advances in precision medicine. He cautioned the audience to recognize the potential limitations of new therapeutic interventions, the duration of time required for incorporation of medical advances into clinical practice, and importance of the breadth of factors (medical, genetics, socio-economic, environmental) that impact an individual's health and well-being. Finally, he emphasized the importance of the cancer care process which must include careful consideration of patient's wishes.

\section{Conclusion}

Precision medicine is being developed within a complex landscape of public policy, science, economics, law, and regulation. In these and other policy areas, the goal of developing individually-tailored therapies poses novel challenges for health care research, delivery and policy. In this symposium, a range of experts in genetics, medicine, bioinformatics, intellectual property, health economics and bioethics identified and discussed many of the pressing questions raised by the development and practice of precision medicine. These and other issues will need to be taken into account as precision medicine moves ahead and becomes the standard of medical practice and care in the United States and around the world. 


\title{
Appendix
}

\section{FRONTIERS IN PRECISION MEDICINE II: CANCER, BIG DATA AND THE PUBLIC}

\author{
December 1-2, 2016 \\ University of Utah S.J. Quinney College of Law
}

Agenda

Thursday, December 1, 2016

\section{Tackling Cancer with Precision Data}

\section{a. The Science of Precision Oncology}

Moderator: Ana Maria Lopez, M.D., M.P.H., F.A.C.P., Professor of Internal Medicine; Associate Vice President for Health Equity and Inclusion; and Director of Cancer Health Equity at Huntsman Cancer Institute, University of Utah

Kathleen Cooney, M.D., F.A.C.P., Professor and Chair of Internal Medicine, University of Utah

Scott Tomlins, M.D., Assistant Professor of Genitourinary Pathology, University of Michigan

\section{b. The Challenges of Big Data}

Moderator: Kensaku Kawamoto, M.H.S., M.D., Ph.D., University of Utah

Samir Courdy, M.B.A., Huntsman Cancer Institute

Rakesh Nagarajan, M.D., Ph.D., Founder and Chief Information Officer at PierianDx and Adjunct Associate Professor of Pathology and Immunology, Washington University in St. Louis

David Nix, Ph.D., Co-Director of Bioinformatics Shared Resource, Huntsman Cancer Institute

\section{c. Precision Prevention}

Moderator: Cornelia Ulrich, M.S., Ph.D., Senior Director of Population Sciences at Huntsman Cancer Institute and Division Chief of Population Health Sciences, University of Utah

Ulrike Peters, M.P.H., Ph.D., M.S., Research Professor of Epidemiology, University of Washington, Full Member at Fred Hutchinson Cancer Research Center 
Marc Lenburg, Ph.D., Professor of Medicine in the Division of Computational Biomedicine, Boston University

\section{Keynote Address}

\section{Catalyzing Translational Innovation}

Christopher Austin, M.D., Director, National Center for Advancing Translational Sciences (NCATS)

\section{The Public and Precision Medicine}

\section{a. Recruiting Large Research Cohorts}

Moderator: James Tabery, Ph.D., Professor of History and Philosophy of Science, University of Utah

Laurence Meyer, M.D., Ph.D., Veterans Administration Medical Center

Catherine Schaefer, Ph.D., Kaiser Permanente Division of Research

\section{b. Engaging the Public in the Program}

Moderator: Jeffrey Botkin, M.D., M.P.H., Professor of Pediatrics, University of Utah

Angela Fagerlin, Ph.D., Professor of Population Health Sciences, University of Utah

Kimberly Kaphingst, Sc.D., Health Communication Researcher, University of Utah

\section{c. The Ethics of Access and Precision Medicine}

Moderator: Leslie Francis, Ph.D., J.D., Professor of Law and Philosophy, University of Utah

Jessica Roberts, J.D., Director of the Health Law and Policy Institute, University of Houston Law Center

Maya Sabatello, Ph.D., Columbia University College of Physicians and Surgeons

\section{Friday, December 2, 2016}

\section{Economics and Precision Medicine}

\section{a. Patenting Precision Medicine}

Moderator: Jorge L. Contreras, J.D., Professor of Law, University of Utah

Bernard Chao, J.D., Associate Professor of Law, University of Denver

Colleen Chien, J.D., Associate Professor of Law, Santa Clara University

Shubha Ghosh, J.D., Ph.D., Director of the Technology Commercialization Law Center, Professor of Law, Syracuse University

Kshitj Kumar Singh, Ph.D., Assistant Professor of Law, Amity Institute of Advanced Legal Studies 


\section{b. Providers, Payer, and Laboratory Testing}

Moderator: Allie Grossmann, M.D., Ph.D., Assistant Professor of Pathology, University of Utah

Diana Brixner, R.Ph., Ph.D., Professor in the Department of Pharmacology, University of Utah

Christopher Corless, M.D., Ph.D., Professor of Pathology, Oregon Health \& Science University

Craig Nichols, M.D., Intermountain Healthcare

\section{Concluding Remarks}

Willard Dere, M.D., Professor of Internal Medicine, University of Utah Jorge L. Contreras, J.D., Professor of Law, University of Utah 